\title{
Transglutaminase 2 Regulates Self-renewal and Stem Cell Marker of Human Colorectal Cancer Stem Cells
}

\author{
SANGHEE KANG ${ }^{1}$, SANG CHEUL OH ${ }^{2,3}$, BYUNG WOOK MIN ${ }^{1}$ and DAE-HEE LEE ${ }^{2,3}$ \\ ${ }^{1}$ Department of Surgery, Korea University Guro Hospital, Seoul, Republic of Korea; \\ ${ }^{2}$ Department of Oncology, Korea University Guro Hospital, Seoul, Republic of Korea; \\ ${ }^{3}$ Graduate School of Medicine, Korea University College of Medicine, Seoul, Republic of Korea
}

\begin{abstract}
Background/Aim: The aim of this study was to investigate the role of transglutaminase 2 (TGM2) in colorectal cancer stem cells (CCSCs). Materials and Methods: We used the TU12 cell line possessing CD133expressing CCSCs. After isolating CD133 (-) and CD133 (+) CCSCs, we overexpressed and knocked-down TGM2 to investigate its role in human CCSCs. Results: The expression level of TGM2 was 25-fold higher in tumorigenic cells than non-tumorigenic cells. We found that knockdown of TGM2 by specific RNA interference markedly inhibited cell growth and caused down-regulation of the stemness markers, CD133, SOX2, and $\beta$-catenin. We further demonstrated that knockdown of TGM2 inhibited cell metastatic abilities by down-regulating $N$-cadherin and vimentin and up-regulating E-cadherin. These findings revealed that TGM2 expression is markedly increased in human colorectal cancer and that down-regulation of TGM2 in tumors may serve as a treatment for colorectal cancer patients. Therefore, this study indicate that TGM2 affects the metastatic potential and stemness of CCSCs by regulating EMT- and stemness-related proteins. Conclusion: The metastatic potential of CSCs arises from highly expressed TGM2.
\end{abstract}

Colorectal cancer (CRC) is one of the common cancers worldwide. Despite the recent advances in the cancer therapy with chemotherapy, radiation, and surgery, there are still problems with metastasis to other organs such as lymph nodes, liver, and lungs. A better understanding of the pathways of metastasis mechanisms will improve the

Correspondence to: Dae-Hee Lee, Department of Oncology, Korea University Guro Hospital, Seoul, Republic of Korea; Byung Wook Min, Department of Surgery, Korea University Guro Hospital, Seoul, Republic of Korea. Tel: +82 226261998, Fax: +82 226261993, e-mail: neogene@korea.ac.kr; gsmin@korea.ac.kr

Key Words: TGM2, CCSC, stemness, EMT. management of CRC. The less effective traditional anticancer therapies have been attributed to the existence of highly drug-resistant or slow proliferating cells with stemlike properties, which are cancer stem cells (CSCs). CSCs have the ability to induce carcinogenesis and metastasis (1, 2). In addition, CSC-associated drug resistance is considered to be a main reason for recurrence. Recently, CSCs have emerged as playing a role in inhibiting cancer growth. CRC also has a CSC population. Some of the CSCs express CD133, which is well studied in brain tumors. In brain tumors, CD133 is a marker of tumor-initiating cells and is expressed in approximately $7 \%$ of the total tumor population. Furthermore, recent studies showed that the epithelial mesenchymal transition (EMT) and stemness are closely bounded to CSCs including human glioblastoma (3).

Transglutaminase 2 (TGM2) is the most ubiquitously expressed protein in the transglutaminase family, which catalyzes the cross-linking of proteins $(4,5)$. TGM2 has been suggested to be involved in tumorigenesis during physiological processes in numerous cancers (6-8).

In the present study, we hypothesized that TGM2 may play a role in the invasion, migration, and stemness properties of CCSCs. We used the TU12 cell line possessing CD133 expressing CCSCs. After isolating CD133 (-) and CD133 (+) CCSCs, we overexpressed and knocked down TGM2 to investigate its role in human CCSCs. Herein, we report that TGM2 regulates EMT- and stemness-related proteins in TU12 human CCSCs.

\section{Materials and Methods}

Cell culture. Human CCSCs were established from acutely resected human tumor tissues (9). Tumor-sphere cultures were performed as described previously, with some modifications, and grown in Dulbecco's modified Eagle's medium-F12 (GibcoInvitrogen, La Jolla, CA, USA) containing penicillin G, streptomycin sulfate, B-27 (Gibco-Invitrogen, La Jolla, CA, USA), and recombinant human EGF and FGF $(20 \mathrm{ng} / \mathrm{ml}$; R\&D Systems, Minneapolis, MN, USA). Cells were cultured at $37^{\circ} \mathrm{C}$, with $95 \%$ relative humidity, and $5 \% \mathrm{CO}_{2}$. 
Table I. The primers used for PCR analysis.

\begin{tabular}{lll}
\hline Gene & Forward primer (5'-3') & Reverse primer (5'-3') \\
\hline E-cadherin & TTC CCT GCG TAT ACC CTG GT & GCG AAG ATA CCG GGG GAC ACT CAT GAG \\
Snail & ACTATGCCGCGCTCTTTCCT & AGTCCTGTGGGGCTGATGTG \\
Vimentin & TCCAGCAGCTTCCTGTAGGT & CCCTCACCTGTGAAGTGGAT \\
GAPDH & CA AGG CTG AGA ACG GAA G & AGA GGG GGC AGA GAT GAT GA \\
$\beta$-actin & GTC TTC CCC TCC ATC GTG & AGG TGT GGT GCC AGA TTT TC \\
\hline
\end{tabular}

Magnetic Cell Sorting (MACS). Cells were labeled with primary CD133/1-PE antibody (Miltenyi Biotec, Bergisch Gladbach, Germany), then labeled with anti-PE microbeads (Miltenyi Biotec, Bergisch Gladbach, Germany) and isolated on a MACS LS column (Miltenyi Biotec, Bergisch Gladbach, Germany). All procedures were performed according to the manufacturer's instructions. Isotypematched mouse immunoglobulins (Miltenyi Biotec, Bergisch Gladbach, Germany) served as controls. The purity of sorted cells was evaluated by flow cytometry and Western blotting. Flow cytometry was done using a BD FACSCalibur. Data were analyzed by BD FACSCalibur software, which is provided with the system.

$R N A$ isolation and RT-PCR. Total RNA was isolated, and cDNA was synthesized from $1 \mu \mathrm{g}$ of total RNA using the Superscript II system (Invitrogen, Carlsbad, CA, USA) in accordance with the manufacturer's instructions. For all RT-PCR analysis, $\beta$-actin and GAPDH were used as loading controls. The primers used in this study are listed in Table I. The data of RT-PCR were quantified by ImageJ software (National Institute of Health, USA).

Western blotting. Total protein lysates $(50 \mu \mathrm{g})$ were resolved on an SDS-PAGE gel, transferred onto PVDF membranes, and then immunoblotted with mouse anti-TGM2 (Abcam, Cambridge, MA, USA), mouse anti-CD133 (Miltenyi Biotec, Bergisch Gladbach, Germany), goat anti-SOX2 (Santa Cruz Biotechnology, Inc. Dallas, TX, USA), rabbit anti-E-cadherin (Santa Cruz Biotechnology, Inc., Dallas, TX, USA), goat anti-vimentin (Santa Cruz Biotechnology, Inc., Dallas, TX, USA), or rabbit anti-snail (Santa Cruz Biotechnology, Inc., Dallas, TX, USA). The membranes were incubated with horseradish peroxidase-conjugated secondary antibody. $\beta$-actin was used as a loading control. The data of western blotting were quantified by ImageJ software (National Institute of Health, USA).

Immunofluorescence. Cells grown on glass coverslips were fixed with $3.7 \%$ formaldehyde for $15 \mathrm{~min}$, followed by permeabilization with $0.5 \%$ Triton X-100 for 15 min at room temperature. Cells were then blocked for $1 \mathrm{~h}$ with $3 \%$ bovine serum albumin. Primary antibodies were applied overnight at $4{ }^{\circ} \mathrm{C}$, followed by incubation with Alexa fluor-594-conjugated secondary antibody (Molecular Probes, Eugene, OR, USA) or FITC-conjugated secondary antibody (Sigma, St. Louis, Missouri, USA). The nuclei were co-stained with 4', 6-diamidino-2-phenylindole (DAPI) and visualized with fluorescence microscopy.

TGM2 cDNA transfection. A TGM2 full-length cDNA (NM_004613) clone was purchased by OriGene USA, Inc (Rockville, MD, USA). Cells were transfected with plasmid harboring TGM2 (pCDNA/TGM2) or with vector (pCDNA) only, using LipofectAMINE 2000 (Life Technologies, Carlsbad, CA, USA). Before transfection, the cells were plated on 6-well plates in antibiotic-free serum. At the time of transfection, the cell confluence was $70-80 \%$. All procedures were performed according to the manufacturer's instructions. The cells were incubated at $37^{\circ} \mathrm{C}$ in a humidified atmosphere with $5 \% \mathrm{CO}_{2}$ for $48 \mathrm{~h}$. After $48 \mathrm{~h}$, the cells were evaluated by western blotting or used in the migration, invasion, and sphere formation assays.

TGM2 shRNA transfection and selection. TGM2 shRNA (SC-37514V), control shRNA (SC-108080), and transduction reagent were purchased from Santa Cruz Biotechnology, Inc (Dallas, TX, USA). FACS-sorted CD133(-) and CD133(+) TU12 cells were plated on a 6 -well plate in antibiotic-free medium. The cells were grown to 70-80\% confluence and transfected with TGM2 shRNA and control shRNA using lentiviral particles. After $48 \mathrm{~h}$ of incubation with shRNA, the cells were selected using puromycin $(1 \mu \mathrm{g} / \mathrm{ml})$ for 7 days.

Sphere-forming assays. Survival of the human CCSCs in response to TGM2 was assessed by sphere formation. For this assay, human CCSCs, with or without TGM2, were seeded at 1,000 cells per well in a 24-well plate and cultured for 5 days.

Migration and invasion assay. Sorted cells transfected with the TGM2 plasmid, vector plasmid, TGM2 shRNA, or control shRNA were seeded at a density of $2.5 \times 10^{4}$ cells/well onto a 24 -well transwell plate $(8.0 \mu \mathrm{m}$, Corning). For the invasion assay but not the migration assay, the transwell was coated with Matrigel (BD Biosciences, San Jose, CA, USA). All procedures were performed according to the manufacturer's instructions. The migration and invasion assays were performed at $37^{\circ} \mathrm{C}$ in a humidified atmosphere under $5 \% \mathrm{CO}_{2}$ for $48 \mathrm{~h}$ in triplicate. After $48 \mathrm{~h}$, cells were fixed and stained using Giemsa solution and non-migrated and non-invaded cells were removed by cotton swabbing. Migrated and invaded cells were counted.

Statistical analysis. Statistical analysis was performed using GraphPad InStat 7 software (GraphPad Software, Inc., San Diego, CA, USA). Values are reported as the mean \pm SD. $p<0.05$ was considered significant and is indicated by asterisks in the figures.

\section{Results}

TGM2 expression is decreased in human CRC. We first investigated whether expression of TGM2 is overexpressed in human colorectal cancer. Immunohistochemistry (IHC) 
A

Tumor

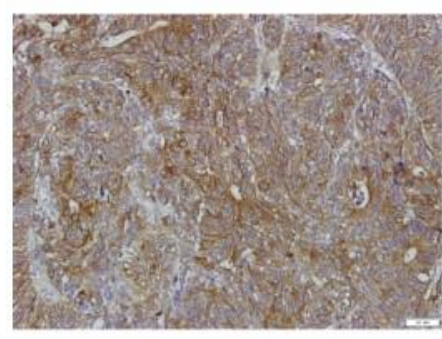

Normal

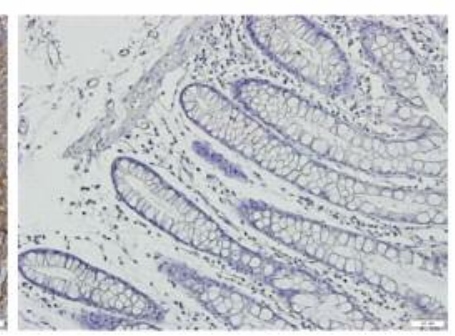

B

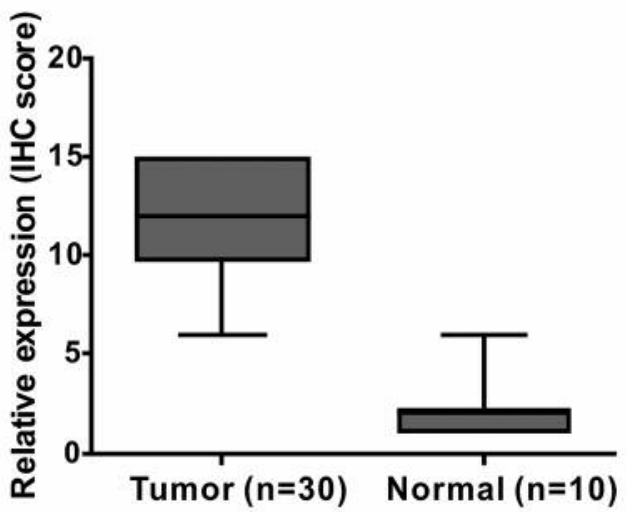

Figure 1. TGM2 expression in tumor-adjacent normal colorectal tissue and CRC tissue. (A) Representative pictures of immunohistochemistry on human CRC specimens stained for TGM2. Magnification: $\times 40 .(B)$ Box plots indicate the percentage of TGM2-positive cells in normal (right box) and tumor samples (left box). ${ }^{* *} p<0.01$ for normal versus tumor tissues. Normal, $n=10$; Tumor, $n=30$. The data for the box plots derive from the immunohistochemical analyses in A.

staining was performed in TMA slide containing normal and tumor cancer tissues. As shown in Figure1A, from the serial sections of paired normal colorectal tissue and tumor of CRC patients, TGM2 was highly expressed in tumors but lost in normal colorectal tissues. Our IHC data showed that there was a significantly higher level of TGM2 in tumors than in normal colon tissues $(p<0.01$, Figure 1B). These results suggested that TGM2 may be an important prognostic marker in CRC patients.

TU12 CCSCs CD133(+) cells possess stemness properties and metastatic potential. The TU12 CCSC line was sorted into CD133 (-) cells and CD133 (+) cells using an antiCD133-PE antibody and MACS. As shown in Figure 2A, CD133 (-) and CD133 (+) cells have different properties with respect to stemness. CD133 (+) cells have higher protein expression of stemness markers (CD133 and SOX2). For comparisons in this study, relative values for CD133 (-) cells were defined as "1". CD133 and SOX2 expression levels in CD133 (+) cells were at least 12-fold and 4-fold higher than those in CD133 (-) cells, respectively $(p<0.001)$. As previously reported (10), TGM2 was significantly upregulated in CD44 (+) cells and in our study, its expression level was at least 3-fold higher than that in CD133 (-) cells $(p<0.001)$ (Figure 2B). A sphere formation assay was conducted to determine the self-renewal ability of TU12 CCSC CD133 (+) cells (Figure 2C). The number of spheres of TU12 CD133 (+) cells was at least 4-fold higher than that of CD133 (-) cells. Since CSCs are responsible for tumor metastasis, we compared the migration and invasion abilities of TU12 CD133 (-) cells and TU12 CD133 (+) cells to estimate the metastatic potential in vitro. As shown in Figure 2D (migration) and 2E (invasion), CD133 (+) cells had an at least 2-fold higher migration ability and 1.5-fold higher invasion ability than CD133 (-) cells ( $\mathrm{p}<0.05$ ). Taken together, these results indicate that TU12 CD133 (+) cells that highly expressed TGM2 were successfully isolated, and these cells had stemness properties and metastatic potential.

TGM2 enhances the self-renewal ability of TU12-derived CSCs. We produced (Figure 3A and B) CD133 (-) and CD133 (+) cells overexpressed or depleted in TU12. CD133 (+) cells highly expressing TGM2 have potent self-renewal ability. We hypothesized that TGM2 is involved in self-renewal potency and performed a sphere formation assay using experimentally manipulated cells (data not show). Increased expression of TGM2 enhanced the sphere formation ability. We also found that TGM2 enhanced the cell growth on CCSCs. These results indicated that TGM2 is directly involved in the self-renewal ability of TU12-derived CSCs.

TGM2 directly regulates stemness- and EMT-related proteins. To investigate the role of TGM2 in the metastatic potential of TU12-derived CSCs, we performed a migration and invasion assay using transwells. Our previous data (Figures 3 and 4) suggest that TGM2 is directly associated with the stemness and metastatic ability of CCSCs. We also further investigated the molecular mechanism underlying these properties. First, we confirmed the expression of stemness proteins by western blotting (Figure 2A) and EMTassociated genes (E-cadherin, vimentin, and snail) by RTPCR (Figure 4A and Table I). For comparisons, relative values for CD133 (-) cells were considered to be "1". Compared to CD133 (-) cells, E-cadherin, epithelial marker, was down-regulated by half $(0.36 \pm 0.02)$ in CD133 (+) cells. Mesenchymal marker was up-regulated in CD133 (+) cells; 
A

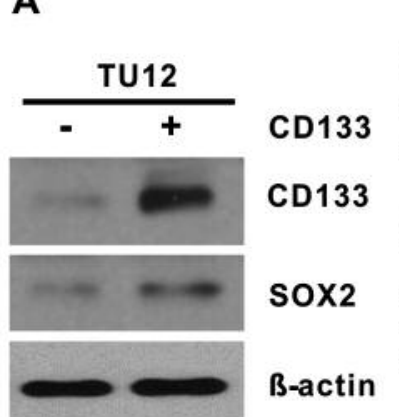

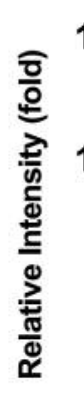

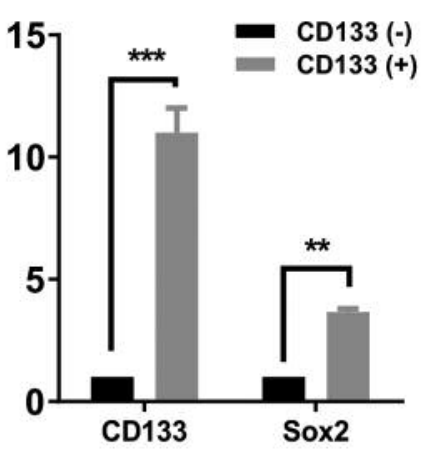

B
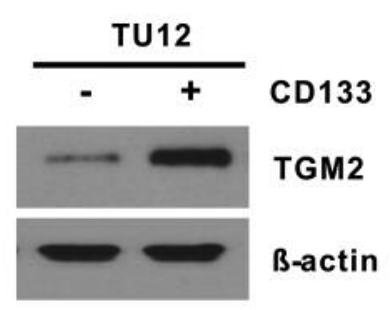

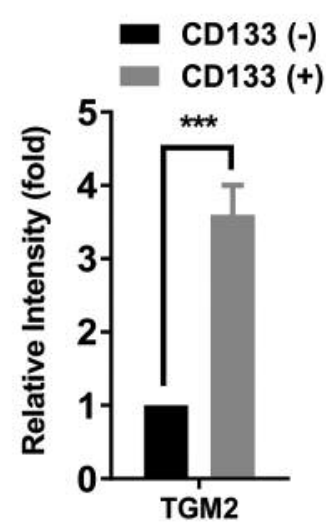

C

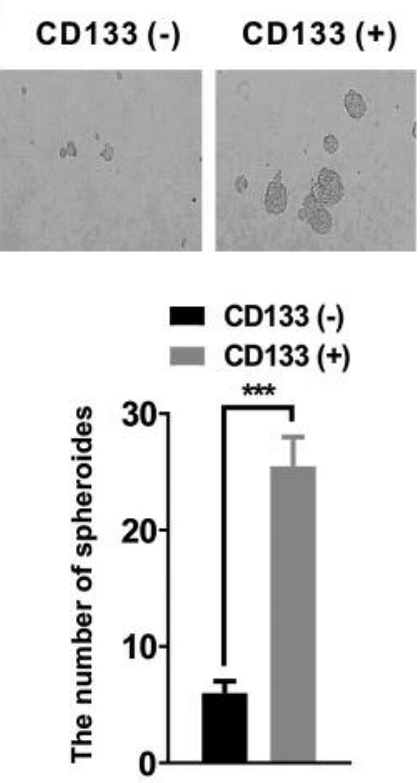

D
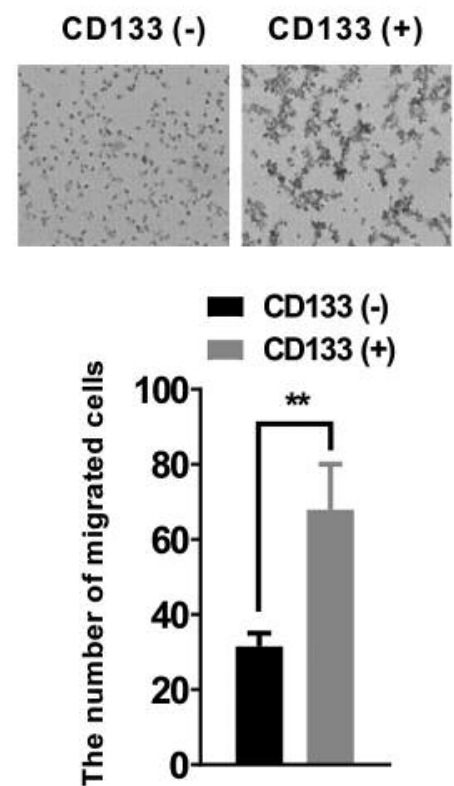

$\mathbf{E}$
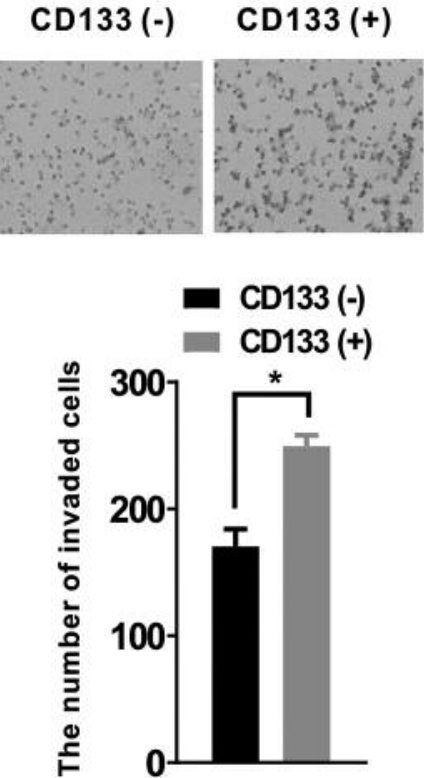

Figure 2. Stemness and metastatic potential of CD133 (-) cells and CD133 (+) cells isolated from a colorectal cancer line. (A) CD133 and SOX2 expression was analyzed in the sorted CD133 (-) and CD133 (+) colorectal cancer CCSCs by western blot. (B) Expression levels of TGM2 in CD133 (-) and CD133 (+) cells were measured by western blot. $\beta$-actin was used as a loading control. (C) Sphere formation assay. Isolated CD133 $(-)$ and CD133 (+) cells were seeded at a density of $1 \times 10^{4}$ cells/well onto 6-well plates, and spheres larger than 100 um were counted at day 5. Scale bar $=100 \mu \mathrm{m}$. (D) Migration assay. The migratory ability of isolated cells was assessed in 24-well transwell plates with $8 \mu \mathrm{m}$ filters. (E) Invasion assay. The invasive ability of CD133 (-) and CD133 (+) cells was determined in 24-well transwell plates with 8 um filters coated with Matrigel. Images were obtained at 40x magnification. Data are expressed as the mean \pm standard error of the mean (SEM) of three independent experiments performed $(* p<0.05, * * p<0.01, * * * p<0.001)$.

vimentin was up-regulated by at least 3 -fold and snail was up-regulated by 2-fold compared to CD133 (-) cells $(p<0.001)$ (Figure 4B). Because TU12 CD133 (+) cells highly expressing TGM2 have strong stemness and metastatic properties, we investigated whether TGM2 affects stemness and EMT-related protein expression by using TGM2-overexpressing and TGM2-knockdown cells.

First, the stemness-related proteins CD133 and Sox-2 were up-regulated in TGM2-overexpressing CD133 (+) cells
(Figure 4C) and down-regulated in TGM2-knockdown CD133 (+) cells (Figure 4). However, in CD133 (-) cells, TGM2 did not affect CD133 expression. Next, EMT-related markers' expression was investigated. The epithelial marker, E-cadherin was down-regulated in control CD133 (+) cells and up-regulated in control CD133 (-) cells (Figure 4C) as reported in previous studies. When TGM2 was overexpressed, expression level of E-cadherin was not influenced in both CD133 (-) and CD133 (+) cells (Figure 
A

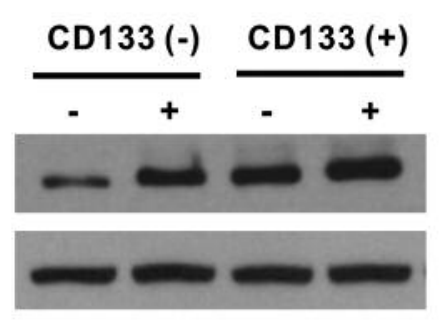

pcDNA-TGM2

TGM2

ß-actin
C

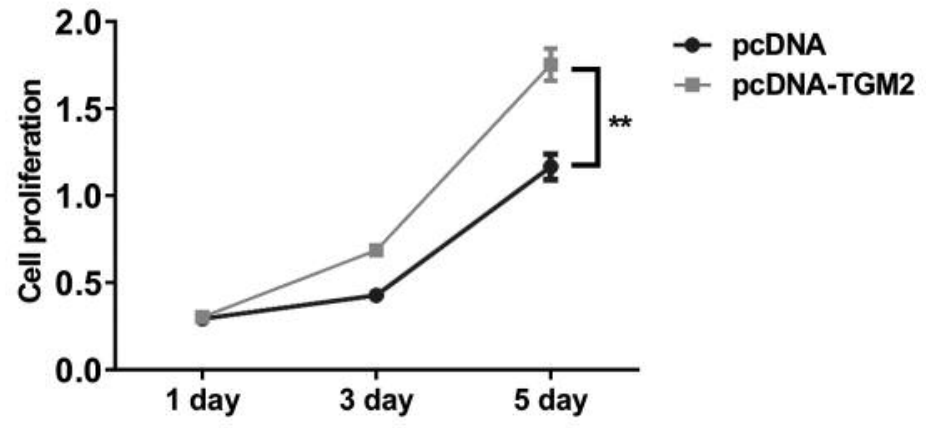

D

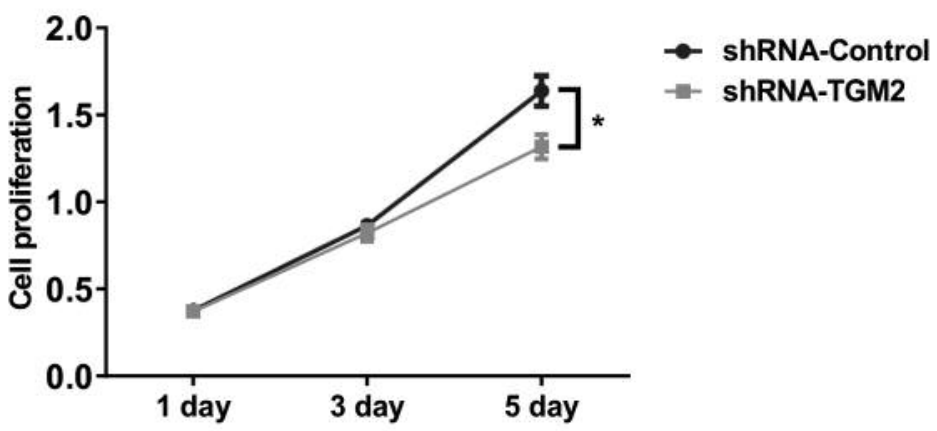

Figure 3. Effect of TGM2 on the cell growth ability of colorectal cancer CCSC CD133 (-) and CD133 (+) cells. (A) Western blot of TGM2overexpressing colorectal cancer CCSC CD133 (-) and CD133 (+) cells by using anti-TGM2 antibody. pCDNA and pCDNA-TGM2 indicates empty vector-transfected cells and TGM2 cDNA-transfected cells, respectively. (B) Western blot of TGM2-knockdown Colorectal cancer CCSC CD133 (-) and CD133 (+) cells by using an anti-TGM2 antibody. $\beta$-actin was used as a loading control. $(C$ and $D)$ Cell growth ability of TGM2-overexpressing colorectal cancer CCSC CD133 (-) and CD133 (+) cells. Empty vector-transfected cells ( $p C D N A)$ served as a negative control. Cell growth ability of TGM2-knockdown colorectal cancer CCSC CD133 (-) and CD133 (+) cells. Control shRNA-transfected cells served as a negative control. Data are expressed as the mean \pm standard error of the mean (SEM) of three independent experiments performed $\left(* p<0.05, *^{*} p<0.01\right)$.

4C). However, when TGM2 was knocked down, expression of E-cadherin was increased in CD133 (-) and CD133 (+) cells (Figure 4D). The mesenchymal marker, snail was down-regulated in CD133 (-) cells and up-regulated in CD133 (+) cells in this study (Figure 4C). Snail expression was significantly suppressed by TGM2- knockdown (Figure 4D). These results indicated TGM2 directly regulated stemness- and EMT-related proteins (Figure 5).

\section{Discussion}

Traditional therapy targets rapidly dividing tumor cells for the maximum reduction of tumor; however, the tumorinitiating cells (TICs) or cancer stem cells (CSCs) can migrate to other organs and provide a high possibility of recurrence (11-14). Therefore, targeting CSCs is an important strategy to cure metastatic cancer, which is resistant to conventional therapy. However, the mechanism of the metastatic potential and chemoresistance of CCSCs is unclear. This study shows that TGM2 is an important molecule for tumor initiation and regulation of metastasis. The metastatic potential and stemness properties of CSCs are derived from elevated levels of TGM2. In the present study, we isolated CD133 (+) cells from the human CCSC line, TU12. TU12 CD133 (+) cells had a distinctive expression pattern of stemness and EMT markers compared to CD133 (-) cells (Figures 2A and 4A). In addition, TU12-derived CCSCs possessed enhanced self-renewal, migratory, and invasive ability. CD133 is a representative stemness marker in CCSCs and plays a role in the self-renewal ability of CCSCs. Because TGM2 is up-regulated in CSCs and involved in cell motility as an actin-binding protein (15), we speculated that TGM2 is involved in the stemness phenotype and metastatic ability of CSCs. Our results coincided with previous findings. Overexpression of TGM2 increased the self-renewal and metastatic ability (migration and invasion) of CSCs, whereas suppression of its expression remarkably decreased these abilities (Figures 3 and 4). 
A

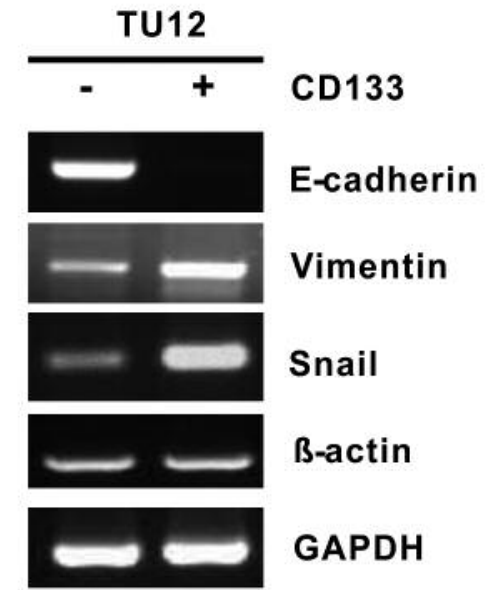

B

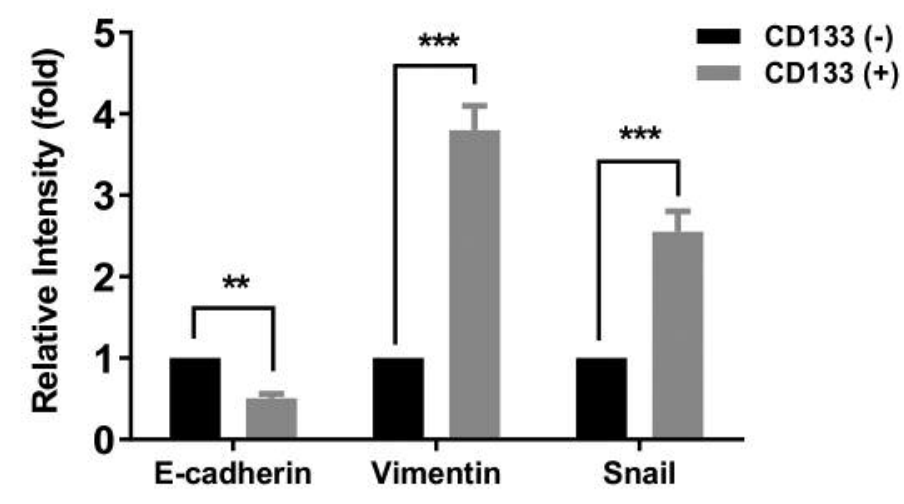

D

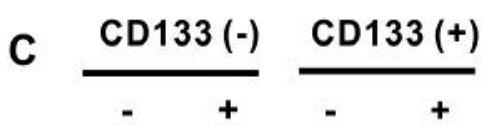

pcDNA-TGM2

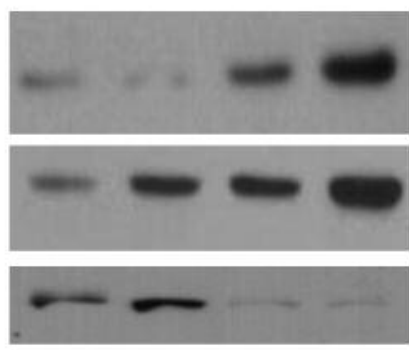

CD133

Sox2

E-cadherin

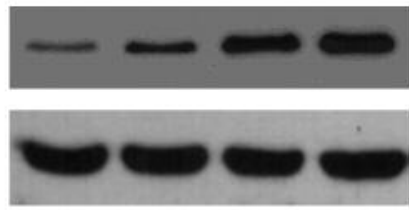

Snail

B-actin
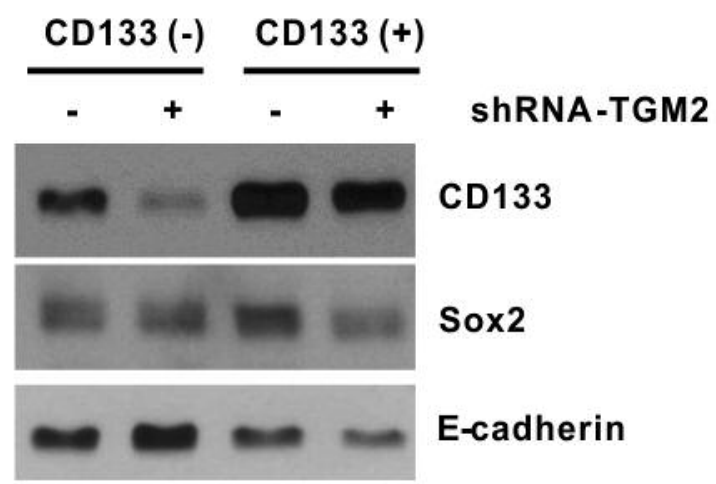

E-cadherin

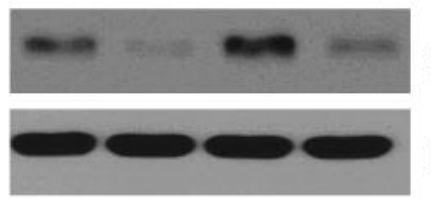

Snail

\section{B-actin}

Figure 4. Effect of TGM2 on expression of stemness and EMT markers in colorectal cancer CCSC CD133 (-) and CD133 (+) cells. (A) The expression levels of EMT markers in CD133 (-) and CD133 (+) cells were measured by RT-PCR. (B) Relative intensity difference between CD133 $(-)$ and CD133 (+) cells. GAPDH and $\beta$-actin were used as loading controls. Error bars represent the mean \pm SEM $(* p<0.05$, * $p<0.01$, $* * * p<0.001)$. (C) and (D) Expression patterns of stemness (CD133 and SOX2) and EMT markers (E-cadherin and snail) in TGM2-overexpressing (C) and TGM2-knockdown (D) Colorectal cancer CCSC CD133 (-) and CD133 (+) cells. $\beta$-actin was used as a loading control.

In addition, the expression of stemness proteins (CD133 and SOX2) and EMT proteins (E-cadherin and snail) was directly regulated by TGM2. Overexpression and depletion (Figure 4) of TGM2 enhanced and suppressed CD133 expression of CSCs, respectively. These results agreed with the sphere formation assay results. EMT is a unique process initially occurring in embryonic development in which cells lose epithelial features and gain mesenchymal properties. In epithelial cancers, metastasis is thought to occur by EMT. Epithelial cells are detached from the basal membrane and they are then more capable of migrating to other sites or they become more invasive and enter the blood and lymphatic system. Loss of E-cadherin is a critical step in EMT. With the loss of E-cadherin expression, epithelial cells break down cell-cell adhesions and become more migratory (16-19). Overexpression of TGM2 did not influence E-cadherin expression in both CD133 (-) and CD133 (+) cells (Figure 4B). Thus, in TGM2- transfected CD133 (+) cells, enhanced metastatic ability was due to TGM2. However, knockdown of TGM2increased the expression of E-cadherin in CD133 (-) and CD133 (+) (Figure 4C). This result agreed with the attenuated migration and invasion ability. $\beta$-catenin is also known as another EMT marker that is up-regulated during EMT and as described above, $\beta$-catenin was regulated by TGM2. However, in CD133 (-) cells, increased $\beta$-catenin by TGM2 was not parallel with metastatic ability. This 


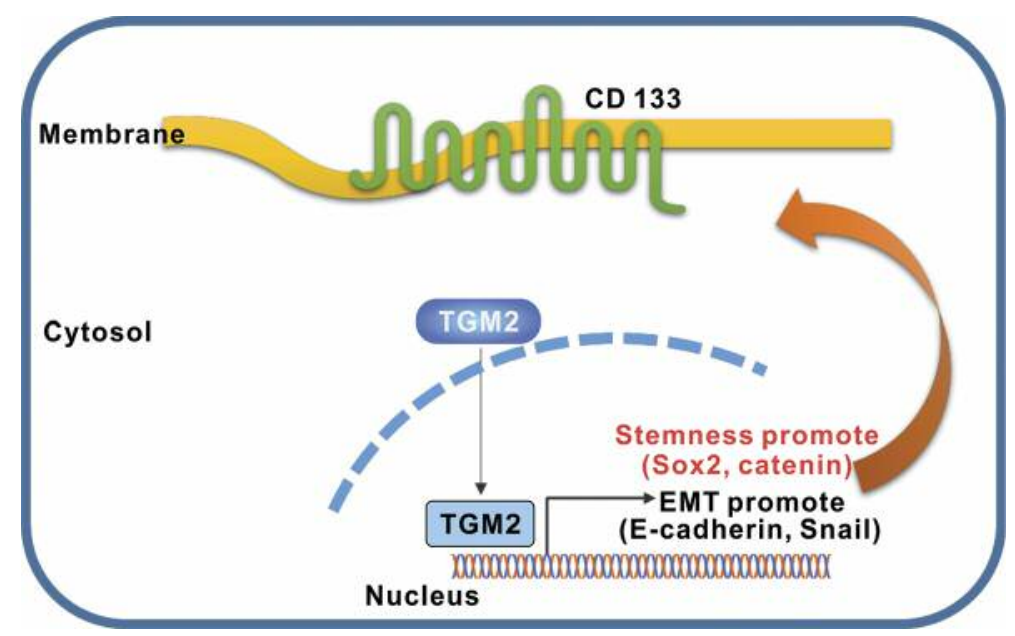

Figure 5. Graphical summary of the effect of TGM2 on colon cancer cells. TGM2 directly regulated stemness- and EMT-related proteins in colon cancer cells.

discrepancy is due to high levels of E-cadherin. Taken together, TGM2 directly affects stemness, migration, and invasion by regulating stemness- and EMT-related proteins.

Recent reports suggest that EMT is closely related to stemness properties in CSCs. The EMT generates cells with properties of stem cells, as the induction of an EMT (i.e. expression of TGF- $\beta$, twist, and snail) in immortalized human mammary epithelial cells acquired mesenchymal traits and the expression of stem-cell markers $(20,21)$. Our study shows similar results in that stemness and EMT were induced at the same time in TU12-derived CSCs with upregulation of TGM2. In addition, overexpression of TGM2 also induced the EMT and CSC phenotype in human TU12 CRCs. Therefore, stemness and the EMT are closely associated through TGM2-related pathway, and TGM2 may play a role as an EMT and stemness marker in TU12 human colorectal cancer cells.

In summary, this study demonstrated that TGM2 promotes CCSCs survival by modulating EMT through the selfrenewal pathway. Thus, TGM2 may be suitable as a prognostic marker for metastatic cancer and cancer resistant to conventional therapy, and it may also be a potential therapeutic target to prevent tumor formation and metastasis.

\section{Conflicts of Interest}

No potential conflict of interest was reported by the authors.

\section{Acknowledgements}

This work was supported by a Grant from the National Research Foundation (NRF) of Korea funded by the Korean government (MSIP) (NRF-2015R1D1A1A01058303) and the Grant of the Korea Health Technology R\&D Project through the Korea Health Industry
Development Institute (KHIDI), funded by the Ministry of Health \& Welfare, Republic of Korea (grant number: HI15C1665).

\section{References}

1 Li P, Zhou C, Xu L and Xiao H: Hypoxia enhances stemness of cancer stem cells in glioblastoma: An in vitro study. Int J Med Sci 10(4): 399-407, 2013.

2 Karamboulas C and Ailles L: Developmental signaling pathways in cancer stem cells of solid tumors. Biochim Biophys Acta 1830(2): 2481-2495, 2013.

3 Zarkoob H, Taube JH, Singh SK, Mani SA and Kohandel M: Investigating the link between molecular subtypes of glioblastoma, epithelial-mesenchymal transition, and cd133 cell surface protein. PLoS One 8(5): e64169, 2013.

4 Eckert RL, Kaartinen MT, Nurminskaya M, Belkin AM, Colak G, Johnson GV and Mehta K: Transglutaminase regulation of cell function. Physiol Rev 94(2): 383-417, 2014.

5 Ballestar E, Abad C and Franco L: Core histones are glutaminyl substrates for tissue transglutaminase. J Biol Chem 271(31): 18817-18824, 1996.

6 Shrestha R, Tatsukawa H, Shrestha R, Ishibashi N, Matsuura T, Kagechika H, Kose S, Hitomi K, Imamoto N and Kojima S: Molecular mechanism by which acyclic retinoid induces nuclear localization of transglutaminase 2 in human hepatocellular carcinoma cells. Cell Death Dis 6: e2002, 2015.

7 Kang JH, Lee SH and Kim SY: Discovery of a novel target for renal cell carcinoma: Transglutaminase 2. Cell Death Dis 7: e2200, 2016.

8 Yeo SY, Itahana Y, Guo AK, Han R, Iwamoto K, Nguyen HT, Bao Y, Kleiber K, Wu YJ, Bay BH, Voorhoeve $\mathrm{M}$ and Itahana $\mathrm{K}$ : Transglutaminase 2 contributes to a tp53-induced autophagy program to prevent oncogenic transformation. Elife 5: e07101, 2016.

9 Soeda A, Inagaki A, Oka N, Ikegame Y, Aoki H, Yoshimura S, Nakashima S, Kunisada T and Iwama T: Epidermal growth factor plays a crucial role in mitogenic regulation of human brain tumor stem cells. J Biol Chem 283(16): 10958-10966, 2008. 
10 Fu J, Yang QY, Sai K, Chen FR, Pang JC, Ng HK, Kwan AL and Chen ZP: Tgm2 inhibition attenuates id1 expression in cd44high glioma-initiating cells. Neuro Oncol 15(10): 1353-1365, 2013.

11 Al-Hajj M, Wicha MS, Benito-Hernandez A, Morrison SJ and Clarke MF: Prospective identification of tumorigenic breast cancer cells. Proc Natl Acad Sci USA 100(7): 3983-3988, 2003.

12 Jensen SS, Meyer M, Petterson SA, Halle B, Rosager AM, Aaberg-Jessen C, Thomassen M, Burton M, Kruse TA and Kristensen BW: Establishment and characterization of a tumor stem cell-based glioblastoma invasion model. PLoS One 11(7): e0159746, 2016.

13 Caspani EM, Echevarria D, Rottner K and Small JV: Live imaging of glioblastoma cells in brain tissue shows requirement of actin bundles for migration. Neuron Glia Biol 2(2): 105-114, 2006.

14 Eyupoglu IY, Hahnen E, Heckel A, Siebzehnrubl FA, Buslei R, Fahlbusch R and Blumcke I: Malignant glioma-induced neuronal cell death in an organotypic glioma invasion model. Technical note. J Neurosurg 102(4): 738-744, 2005.

15 Agnihotri N, Kumar S and Mehta K: Tissue transglutaminase as a central mediator in inflammation-induced progression of breast cancer. Breast Cancer Res 15(1): 202, 2013.
16 Radisky DC: Epithelial-mesenchymal transition. J Cell Sci 118(Pt 19): 4325-4326, 2005

17 Yang J and Weinberg RA: Epithelial-mesenchymal transition: At the crossroads of development and tumor metastasis. Dev Cell 14(6): 818-829, 2008.

18 Spano D, Heck C, De Antonellis P, Christofori G and Zollo M: Molecular networks that regulate cancer metastasis. Semin Cancer Biol 22(3): 234-249, 2012.

19 Kaufhold S and Bonavida B: Central role of snaill in the regulation of emt and resistance in cancer: A target for therapeutic intervention. J Exp Clin Cancer Res 33: 62, 2014.

20 Kudo-Saito C, Shirako H, Takeuchi T and Kawakami Y: Cancer metastasis is accelerated through immunosuppression during snail-induced emt of cancer cells. Cancer Cell 15(3): 195-206, 2009.

21 Kang Y and Massague J: Epithelial-mesenchymal transitions: Twist in development and metastasis. Cell 118(3): 277-279, 2004.

Received October 21, 2017

Revised November 26, 2017

Accepted November 27, 2017 